\title{
Eficacia de la moxifloxacina en infecciones odontogénicas
}

\section{Efficacy of moxifloxacin in odontogenic infections}

\author{
Ardila Medina CM*
}

\section{RESUMEN}

Las infecciones de la boca se caracterizan por ser polimicrobianas, endógenas, oportunistas, dinámicas y mixtas debido a que intervienen bacterias aerobias y especialmente anaerobias. La mayor parte de estas infecciones son odontogénicas, siendo las más frecuentes caries, periodontitis, absceso periapical, absceso periodontal, pericoronaritis, pulpitis, sinusitis, osteítis e infección de los espacios aponeuróticos. Las quinolonas presentan una alta penetración tisular y una buena absorción cuando se suministra después de dosis orales, pero solamente los compuestos desarrollados recientemente como la Moxifloxacina demuestran suficiente efectividad clínica y microbiológica. El objetivo de este artículo es presentar la eficacia in vitro e in vivo de la Moxifloxacina para el tratamiento de las infecciones de origen dental.

Palabras clave: Infecciones odontogénicas, tratamiento, antibiótico, moxifloxacina.

\section{SUMMARY}

The infections of the mouth are characterized for being polimicrobians, endogenous, opportunistic, dynamic and mixed because aerobic and specially anaerobic bacteria take part. Most of these infections are odontogenics, being the most frequent decays, periodontitis, periapical abscess, periodontal abscess, pericoronitis, pulpitis, sinusitis, osteitis and infection of the aponeurotic spaces. Quinolonas displays a high tissue penetration and a good absorption when it is provided after oral doses, but only the compounds developed recently as the Moxifloxacin demonstrates sufficient clinical and microbiological effectiveness. The aim of this article is to present the effectiveness in vitro and in vivo of the Moxifloxacin for the treatment of the infections of dental origin.

Key words: Odontogenic infections, treatment, antibiotics, moxifloxacina.

Fecha de recepción: 19 de septiembre de 2008.

Aceptado para publicación: 2 de octubre de 2008.

* Profesor Asistente. Universidad de Antioquía.

Presidente de la Sociedad Colombiana de Periodoncia-Regional. Antioquía.

Candidato a PhD en Epidemiología.

Ardila Medina CM. Eficacia de la moxifloxacina en infecciones odontogénicas. Av. Odontoestomatol 2009; 25 (4): 215-222.

\section{INTRODUCCIÓN}

La cavidad bucal está constituida por una serie de tejidos y estructuras que forman diferentes ecosistemas colonizados por una gran cantidad de microor- ganismos que forman la microbiota oral. Una alteración en este sistema ecológico puede estimular la aparición de enfermedades e infecciones cuya evolución estará condicionada por el estado general de salud del paciente, la causa de la infección, su loca- 
lización y gravedad, así como por el tratamiento instaurado (1). Las infecciones de la boca se caracterizan por ser polimicrobianas, endógenas, oportunistas, dinámicas y mixtas debido a que intervienen bacterias aerobias y especialmente anaerobias $(2,3)$. La mayor parte de estas infecciones son odontogénicas, siendo las más frecuentes caries, periodontitis, absceso periapical, absceso periodontal, pericoronitis, pulpitis, sinusitis, osteítis e infección de los espacios aponeuróticos entre otras. La diseminación de la infección puede comprometer las vías aéreas, involucrar grandes vasos sanguíneos, y de esta forma producir abscesos cerebrales asociados con un gran riesgo de mortalidad (4). En general, las infecciones mixtas constituidas por varias especies bacterianas son típicas en abscesos odontogénicos originados a partir de infecciones apicales (5). La penicilina es aceptada y ampliamente recomendada como el antibiótico de elección, sin embargo, sus elevadas tasas de resistencia antibacteriana requieren monitoreo y atención $(6,7)$. La utilización de la clindamicina (CM) para el tratamiento de abscesos odontogénicos está sustentada en la literatura odontológica $(8,9)$, sin embargo, se ha reportado un incremento en las tasas de resistencia a este antimicrobiano (10, 11). Las quinolonas presentan una alta penetración tisular y una buena absorción cuando se suministra después de dosis orales $(12,13)$, pero solamente los compuestos desarrollados recientemente demuestran suficiente actividad in vitro contra anaerobios $(14,15)$. Es así, como la moxifloxacina (MXF), una fluroquinolona, está siendo utilizada exitosamente en el tratamiento de infecciones respiratorias superiores, sinusitis, infecciones abdominales mixtas, e infecciones de los tejidos blandos (16, 17). Además de su buena penetración tisular, ha demostrado grandes ventajas en el tratamiento de infecciones odontogénicas (18-20) y su perfil de eficacia es comparable con la amoxicilina/ácido clavulánico (AMX-CLA), convirtiéndose en otra alternativa útil para su tratamiento $(18,21)$. El esquema de dosificación de la MXF (400 mg/diarios) podría garantizar un mayor cumplimiento con las prescripciones y su mecanismo de acción también puede proporcionar un alivio más rápido de los síntomas de la infección odontogénica, cuando se compara con la AMX-CLA (22). El objetivo de este artículo es presentar la eficacia in vitro e in vivo de la MXF para el tratamiento de las infecciones de origen dental.

\section{ESTUDIOS IN VITRO}

La tabla 1 presenta los estudios in vitro en los cuales se ha estudiado la efectividad de la moxifloxacina.

Tomás y colaboradores (23), evaluaron los perfiles de susceptibilidad de anaerobios obligados de origen bucal a la MXF y otros antibióticos comúnmente utilizados en odontología. El estudio comprometió 172 anaerobios aislados de la saliva de 43 adultos, determinando concentraciones inhibitorias mínimas (CIM) mediante técnicas de dilución en agar e incubadas bajo condiciones anaeróbicas. Encontraron resistencia a la amoxicilina (AMX) en $45,3 \%$ de los anaerobios obligados y resistencia a la $\mathrm{CM}$ en el $18,6 \%$, concluyendo que los anaerobios obligados patogénicos, oportunistas y no patogénicos presentaron altos porcentajes de resistencia a la AMX y CM y altas CIM para la azitromicina (AZM). La MXF mostró una mayor actividad que la telitromicina (TLM), similar a la detectada a AMX-CLA y metronidazol (MTZ). En consecuencia, la MXF puede representar una posible alternativa antimicrobiana contra anaerobios obligados de origen oral, particularmente en aquellos pacientes con alergia, intolerancia o carencia de respuesta a la AMX-CLA y MTZ.

Isla y colaboradores (24), evaluaron la eficacia de diferentes tratamientos antimicrobianos en infecciones orofaciales utilizando criterios farmacocinéticos y farmacodinámicos. Tras llevar a cabo una revisión bibliográfica que permitió conocer los valores de CIM de cinco de los microorganismos más frecuentemente aislados en infecciones odontogénicas (estreptococos del grupo viridans, Peptostreptococcus spp., Prevotella intermedia, Porphyromonas gingivalis $y$

\section{TABLA 1.- ESTUDIOS IN VITRO EN LOS CUALES SE HA ESTUDIADO LA EFECTIVIDAD DE LA MOXIFLOXACINA}

Anaerobios Obligados

Infecciones Orofaciales

Periodontopatógenos

$(25,30,31)$

Porphyromonas gingivalis

Infecciones Endodónticas 
Fusobacterium nucleatum) y los parámetros farmacocinéticos de 13 antibióticos utilizados en este tipo de infecciones, se realizaron simulaciones farmacocinéticas con parámetros poblacionales medios y se calcularon los índices de eficacia para las 47 pautas posológicas analizadas. Para los antibióticos dependientes de tiempo se calculó el tsupraCIM, mientras que para los dependientes de concentración se determinó el cociente área bajo la curva $(\mathrm{ABC})$ sobre CIM. AMX-CLA (500 mg/8 h o 1.000 mg/12 h) y CM (300 mg/6 h), entre los antibióticos con actividad dependiente de tiempo, y moxifloxacina (400 mg/ $24 \mathrm{~h}$ ) entre los dependientes de concentración mostraron índices de eficacia adecuados frente a los cinco microorganismos considerados como los más frecuentemente implicados en este tipo de infecciones.

En vista del incremento de la resistencia que han presentado los microorganismos a los antibióticos, Mosca y colaboradores (25) determinaron el perfil antimicrobiano de 55 bacterias gram-negativas anaerobias periodontales correlacionadas con infecciones humanas, involucrando 16 cepas de Fusobacterium nucleatum y 39 de Prevotella spp, aisladas de bolsas periodontales de 26 adultos diagnosticados con periodontitis crónica. F. nucleatum fue susceptible a MXF mientras que el $12.5 \%$ de las cepas experimentaron resistencia a AMX y betalactámicos positivos. El $18 \%$ de las cepas de Prevotella spp presentaron resistencia a AMX y betalactámicos positivos y el $7 \%$ lo fueron a la MXF.

Con el fin de evaluar la efectividad in vitro de la MXF y otras quinolonas contra 16 cepas de Porphyromonas gingivalis, Eick y colaboradores (26) realizaron una investigación en donde determinaron las CIM mediante Etest y la técnica de dilución del agar. Su experimento se enfocó principalmente en establecer la tasa de mutación espontánea y la inducción de cepas resistentes. La MXF y la gatifloxacina presentaron las CIM mas bajas, encontrando también que las fluoroquinolonas inducen rápidamente mutaciones, las cuales son dependientes de la cepa y la quinolona. Los autores concluyen que debido a los niveles logrados en el fluido crevicular y a las CIM, la MXF puede prevenir el desarrollo de resistencias y puede ser una alternativa en el tratamiento antibiótico de $P$. gingivalis asociada a periodontitis.
Se evaluó la actividad in vitro de 14 agentes antimicrobianos en presencia de 292 bacterias anaeróbicas obligadas aisladas clínicamente en laboratorios de Santa Mónica (Estados Unidos), Berlín y Leipzig (Alemania), usando una técnica de microdilución (27). Los datos obtenidos a partir de las CIM, sugieren que las nuevas quinolonas como la MXF pueden ser de gran valor en la terapia de infecciones que incluyen bacterias anaeróbicas.

Debido a que los Actinomyces spp se encuentran notablemente asociados a infecciones endodónticas, se realizó una investigación con el fin de determinar la susceptibilidad de Actinomyces naeslundii, Actinomyces gerencseriae, Actinomyces israelii, Actinomyces viscosus, and Actinomyces odontolyticus a la AMX, CM, doxiciclina (DXC), MTZ y MXF, empleando ensayos in vitro (28). La CIM de cada bacteria aislada fue determinada mediante E-test, encontrándose muy buena eficacia de la MXF, mientras que el MTZ no fue activo contra ninguna especie aislada.

Fueron evaluados 21 enterococcus faecalis aislados de canales radiculares de dientes con lesiones periapicales persistentes para susceptibilidad antibiótica a penicilina, AMX, AMX-CLA, eritromicina, AZT, vancomicina, cloranfenicol, tetraciclina, DXC, ciprofloxacina y MXF (29). Las cepas de E faecalis fueron clasificadas como susceptibles o resistentes de acuerdo a los parámetros establecidos por el comité nacional para estándares de laboratorio de los Estados Unidos, encontrándose que fueron susceptibles completamente a AMX-CLA, vancomicina y MXF. La efectividad fue mucho menor en los demás antimicrobianos enumerados anteriormente.

Debido a que las infecciones periodontales con Aggregatibacter actinomycetemcomitans parecen ser refractarias a la terapia convencional, se realizó un estudio con el fin de evaluar la susceptibilidad in vitro de un panel de siete antibióticos administrados oralmente (30). Se examinaron $60 \mathrm{~A}$. actinomycetemcomitans aislados de 43 individuos con periodontitis o gingivitis. Se encontró que el AA fue altamente susceptible a la ciprofloxacina y a la MXF, moderadamente susceptible a la AZT y resistente al MTZ.

Con el fin de evaluar diferencias en la eficacia de antibióticos contra tres patógenos periodontales, se 
realizó un estudio que estableció una biopelícula artificial constituida por $A$. actinomycetemcomitans Y4, Streptococcus constellatus 384b y Porphyromonas gingivalis ATCC 33277 (31). La habilidad de las bacterias para formar una biopelícula variaba. La biopelícula de $S$. constellatus $384 b$ y A. actinomycetemcomitans Y4 contenía mas bacterias viables y mostraba mayor espesor comparada con las de $P$. gingivalis ATCC 33277. Los antibióticos evaluados presentaban diferente eficacia para las distintas especies. La MXF fue el antimicrobiano más eficiente comparado con MTZ, CM y DXC.

Una gran dificultad que debe ser tenida en cuenta en el momento de la selección de antibióticos, mediante pruebas de laboratorio, es que se ha demostrado una mayor resistencia contra las bacterias presentes en la placa subgingival in vivo, comparado con estudios in vitro $(32,33)$.

\section{ESTUDIOS IN VIVO}

Con el fin de estudiar la eficacia del tratamiento profiláctico oral con AMX, CM y MXF en la prevención de bacteriemia después de exodoncias, se evaluaron 221 adultos que requerían extracciones dentales (34). Los antimicrobianos fueron asignados aleatoriamente y fueron administrados una o dos horas antes de iniciar el procedimiento. Muestras sanguíneas de cada uno de los pacientes fueron tomadas a nivel base, 30 segundos, 15 minutos y una hora después de la exodoncia. Se realizó un cultivo e identificación de las bacterias aisladas mediante técnicas microbiológicas convencionales. La profilaxis con AMX y MXF presentó alta eficacia en la reducción de la prevalencia y duración de bacteriemia post extracción, pero la CM no fue efectiva.

Un estudio farmacocinético, prospectivo, multicéntrico, fue realizado para evaluar la efectividad de la MXF en presencia de patógenos que causan sinusitis aguda (35), concluyendo que la MXF oralmente administrada se distribuye con preferencia en los tejidos sinusales alcanzando concentraciones mucho mas altas que en el plasma, demostrando de esta manera su eficacia contra los patógenos involucrados en infecciones sinusales caracterizadas por localizarse en cavidades circunscritas por hueso.
Para comparar la eficacia y seguridad de la MXF con AMX-CLA para el tratamiento de sinusitis bacterianas agudas en adultos se evaluaron 575 pacientes provenientes de países latinoamericanos (36). La tasa de éxito clínico en el grupo que empleó MXF fue 93,4\%, similar al grupo que usó AMXCLA (92,7\%). La erradicación bacteriológica documentada fue del $96,5 \%$ para MXF y del $96,7 \%$ para la AMX-CLA.

Se realizó un ensayo clínico aleatorizado, multicéntrico, fase III, en 475 pacientes adultos con sinusitis aguda que recibieron durante 10 días MXF (400 mg una vez al día) o AMX-CLA (875 mg dos veces al día) (37). La medida primaria eficaz fue la resolución clínica. De los 471 pacientes ingresados en el análisis por intención de tratar (234 MXF, 237 AMX-CLA), el tratamiento con MXF fue equivalente al de la AMXCLA en la prueba de cura ( $85 \%$ vs $82 \%$; IC $95 \%-6 \%$, $13 \%)$. Los autores concluyeron que una dosis diaria de MXF es tan efectiva y segura como dos dosis diarias de AMX-CLA para el tratamiento de sinusitis aguda. Además, al tercer día de tratamiento, los pacientes que ingirieron MXF reportaron sentirse mejor cuando se compararon con el grupo al que se le suministró AMX-CLA $(p<0,02)$.

Warnke y cols. (38), examinaron el espectro de patógenos orales encontrados en abscesos odontogénicos y su susceptibilidad a cinco antimicrobianos. Los resultados in vitro fueron comparados con observaciones clínicas. Se obtuvieron 188 muestras de 94 pacientes con abscesos odontogénicos. Las pruebas de susceptibilidad revelaron una sensibilidad por encima del $99 \%$ para aerobios y del $96 \%$ para anaerobios en el caso de la MXF. Los valores correspondientes para la penicilina fueron inferiores al $61 \%$ y $79 \%$ respectivamente. 92 de los 94 pacientes presentaron una recuperación significativa con el tratamiento descrito.

Un estudio prospectivo, aleatorizado, se realizó en 21 pacientes hospitalizados con abscesos odontogénicos severos con el fin de evaluar el efecto de la MXF comparado con AMX-CLA (18). El resultado clínico primario fue el tiempo empleado hasta que se presentara la remisión clínica, el cual fue en promedio de 6,6 días (rango, 4,3-8,8) para la MXF y de 6 días (rango, 3,8-8,2) para la AMX-CLA. En este 
estudio piloto la MXF presentó resultados promisorios que se comparan con la AMX-CLA.

Pfister y cols. (39), realizaron un ensayo clínico aleatorizado en 92 pacientes con el fin de comparar el uso de MXF y DXC como terapia adjunta al raspado y alisado radicular en pacientes con periodontitis crónica avanzada. Muestras de placa subgingival fueron tomadas de tres sitios con una pérdida de inserción $\geq$ a $5 \mathrm{~mm}$ antes de la terapia mecánica y a los 3, 6 y 12 meses después. Se determinaron Aggregatibacter actinomycetemcomitans, Porphyromonas gingivalis, Tannerella forsythia y Treponema denticola por PCR en tiempo real. Los autores concluyeron que la aplicación adjunta de antimicrobianos reduce la carga de bacterias periodontopatogénicas en periodontitis y que la MXF es mas efectiva que la DXC, convirtiéndose en una alternativa para el tratamiento de casos avanzados de periodontitis crónica.

La tabla 2 presenta los estudios clínicos que demuestran la eficacia de la moxifloxacina en infecciones de origen dental.

\section{CONCLUSIONES}

Es necesario recomendar a los profesionales de la odontología la utilización de pruebas microbiológicas de laboratorio, con el fin de limitar el riesgo de un enfoque terapéutico incorrecto y evitar el abuso de los agentes antimicrobianos.

La MXF puede representar una posible alternativa antimicrobiana contra anaerobios obligados de origen oral, particularmente en aquellos pacientes con

\section{TABLA 2.- ESTUDIOS CLÍNICOS QUEE DEMUESTRAN LA EFICACIA DE LA MOXIFLOXACINA EN INFECCIONES DE ORIGEN DENTAL}

Profilaxis Bacteremia

Sinusitis Aguda . $(35,36,37)$

Abscesos Odontogénicos $(18,38)$

Periodontitis Crónica Avanzada alergia, intolerancia o carencia de respuesta a la AMXCLA y MTZ.

La profilaxis con MXF es una alternativa promisoria para la prevención de bacteriemias post exodoncia cuando no están indicados los antibióticos betalactámicos.

En términos de respuesta clínica y bacteriológica, la MXF fue equivalente a la AMX-CLA para el tratamiento de infecciones odontogénicas y sinusitis bacteriana aguda en adultos.

Debido a las características farmacocinéticas y farmacodinámicas de la $\mathrm{MXF}$, se recomienda como terapia adjunta al raspado y alisado radicular en pacientes con periodontitis crónica severa.

En aquellos casos en que los Actinomyces spp y enterobacterias se encuentran asociados a infecciones endodónticas, el uso de la MXF se convierte en una alternativa eficaz y segura de tratamiento, cuando se presentan este tipo de microorganismos.

La MXF se asocia con un alivio sintomático más rápido cuando se compara con otros antimicrobianos.

\section{BIBLIOGRAFÍA}

1. García Sánchez JE, López R, Prieto J. Antimicrobianos en medicina. Barcelona: Prous Science, 1999;p. 549-57.

2. Rogers AH. Why be down in the mouth? Three decades of research in oral microbiology. Aust Dent J. 2005;50:2-5.

3. Rudney JD, Staikov RK. Simultaneous measurement of the viability, aggregation, and live and dead adherence of Streptococcus crista, Streptococcus mutans and Actinobacillus actinomycetemcomitans in human saliva in relation to indices of caries, dental plaque and periodontal disease. Arch Oral Biol 2002;47:347-59.

4. Rega AJ, Aziz SR, Ziccardi VB. Microbiology and antibiotic sensitivities of head and neck space 
infections of odontogenic origin. J Oral Maxillofac Surg 2006;64:1377-80.

5. Al-Nawas B, Maeurer M. Severe versus Local Odontogenic Bacterial Infections: Comparison of Microbial Isolates. Eur Surg Res 2008;40:22024.

6. Flynn TR, Shanti RM, Levi MH, Adamo AK, Kraut RA, Trieger N. Severe odontogenic infections, part 1: prospective report. J Oral Maxillofac Surg 2006;64:1093-103.

7. Flynn TR, Shanti RM, Hayes C. Severe odontogenic infections, part 2: prospective outcomes study. J Oral Maxillofac Surg. 2006; 64:1104-13.

8. Rush DE, Abdel-Haq N, Zhu JF, Aamar B, Malian $M$. Clindamycin versus Unasyn in the treatment of facial cellulitis of odontogenic origin in children. Clin Pediatr (Phila) 2007;46:154-9.

9. Bratton TA, Jackson DC, Nkungula-Howlett T, Williams CW, Bennett CR. Management of complex multi-space odontogenic infections. J Tenn Dent Assoc. 2002 Fall;82:39-47.

10. Sobottka I, Cachovan G, Stürenburg E, Ahlers MO, Laufs R, Platzer $U$ et al. In vitro activity of moxifloxacin against bacteria isolated from odontogenic abscesses. Antimicrob Agents Chemother 2002;46:4019-21.

11. Boyanova L, Kolarov R, Gergova G, Deliverska E, Madjarov J, Marinov M, Mitov I. Anaerobic bacteria in 118 patients with deep-space head and neck infections from the University Hospital of Maxillofacial Surgery, Sofia, Bulgaria. J Med Microbiol 2006;55(Pt 9):1285-9.

12. Esposito S, Noviello S, D'Errico G, Motta G, Passali D, Aimoni $C$ et al. Concentration of moxifloxacin in plasma and tonsillar tissue after multiple administration in adult patients.J Antimicrob Chemother 2006;57:789-92.

13. Odenholt I, Cars O. Pharmacodynamics of moxifloxacin and levofloxacin against Streptococ- cus pneumoniae, Staphylococcus aureus, Klebsiella pneumoniae and Escherichia coli: simulation of human plasma concentrations after intravenous dosage in an in vitro kinetic model. J Antimicrob Chemother. 2006 Nov;58(5):9605.

14. Schaumann R, Ackermann G, Pless B, Claros MC, Goldstein EJ, Rodloff AC. In vitro activities of fourteen antimicrobial agents against obligately anaerobic bacteria. Int J Antimicrob Agents 2000; 16:225-32.

15. Ackermann G, Schaumann R, Pless B, Claros MC, Goldstein EJ, Rodloff AC.Comparative activity of moxifloxacin in vitro against obligately anaerobic bacteria. Eur J Clin Microbiol Infect Dis 2000; 19:228-32.

16. Petitpretz P, Arvis P, Marel M, Moita J, Urueta J. CAP5 Moxifloxacin Study Group. Oral moxifloxacin vs high-dosage amoxicillin in the treatment of mildto-moderate, community-acquired, suspected pneumococcal pneumonia in adults. Chest 2001; 119:185-95.

17. Patel T, Pearl J, Williams J, Haverstock D, Church D. Efficacy and safety of ten day moxifloxacin $400 \mathrm{mg}$ once daily in the treatment of patients with community-acquired pneumonia. Community Acquired Pneumonia Study Group. Respir Med. 2000;94:97-105.

18. Al-Nawas B, Walter C, Morbach T, Seitner N, Siegel E, Maeurer $M$ et al. Clinical and microbiological efficacy of moxifloxacin versus amoxicillin/ clavulanic acid in severe odontogenic abscesses: a pilot study. Eur J Clin Microbiol Infect Dis. 2008 Jul 29. (Epub ahead of print).

19. Milazzo I, Blandino G, Musumeci R, Nicoletti G, Lo Bue AM, Speciale A. Antibacterial activity of moxifloxacin against periodontal anaerobic pathogens involved in systemic infections. Int $\mathrm{J}$ Antimicrob Agents 2002;20:451-6.

20. Goldstein EJ, Citron DM, Merriam CV, Warren Y, Tyrrel KL, Fernandez $H$. In vitro activities of telithromycin and 10 oral agents against aerobic 
and anaerobic pathogens isolated from antral puncture specimens from patients with sinusitis. Antimicrob Agents Chemother 2003;47:1963-7.

21. Arrieta JR, Galgano AS, Sakano E, Fonseca X, Amábile-Cuevas CF, Hernández-Oliva $G$ et al. Moxifloxacin vs amoxicillin/clavulanate in the treatment of acute sinusitis. Am J Otolaryngol 2007;28:78-82.

22. Rakkar S, Roberts K, Towe BF, Flores SM, Heyd A, Warner J. Moxifloxacin versus amoxicillin clavulanate in the treatment of acute maxillary sinusitis: a primary care experience. Int J Clin Pract 2001;55:309-15.

23. Tomás I, Tomás M, Alvarez M, Velasco D, Potel C, Limeres $\mathrm{J}$ et al. Susceptibility of oral obligate anaerobes to telithromycin, moxifloxacin and a number of commonly used antibacterials. Oral Microbiol Immunol 2007;22:298-303.

24. Isla A, Canut A, Rodríguez-Gascón A, Labora A, Ardanza B, Solinis MA et al. Análisis farmacocinética/farmacodinámica (PK/PD) de la antibioterapia en odontoestomatología. Enferm Infecc Microbiol Clin 2005;23:116-21.

25. Mosca A, Miragliotta L, lodice MA, Abbinante A, Miragliotta G. Antimicrobial profiles of Prevotella spp. and Fusobacterium nucleatum isolated from periodontal infections in a selected area of southern Italy. Int J Antimicrob Agents 2007;30: 521-4.

26. Eick S, Schmitt A, Sachse S, Schmidt KH, Pfister W. In vitro antibacterial activity of fluoroquinolones against Porphyromonas gingivalis strains. J Antimicrob Chemother 2004;54: 553-6.

27. Schaumann R, Ackermann G, Pless B, Claros MC, Goldstein EJ, Rodloff AC. In vitro activities of fourteen antimicrobial agents against obligately anaerobic bacteria. Int J Antimicrob Agents 2000; 16:225-32.

28. LeCorn DW, Vertucci FJ, Rojas MF, ProgulskeFox A, Bélanger $M$. In vitro activity of amoxicillin, clindamycin, doxycycline, metronidazole, and moxifloxacin against oral Actinomyces. J Endod 2007;33:557-60.

29. Pinheiro ET, Gomes BP, Drucker DB, Zaia AA, Ferraz CC, Souza-Filho FJ. Antimicrobial susceptibility of Enterococcus faecalis isolated from canals of root filled teeth with periapical lesions. Int Endod J 2004;37:756-63.

30. Müller HP, Holderrieth S, Burkhardt U, Höffler U. In vitro antimicrobial susceptibility of oral strains of Actinobacillus actinomycetemcomitans to seven antibiotics. J Clin Periodontol 2002;29:736-42.

31. Eick S, Seltmann T, Pfister W. Efficacy of antibiotics to strains of periodontopathogenic bacteria within a single species biofilm - an in vitro study. J Clin Periodontol 2004;31:376-83.

32. Goncalves MO, Coutinho-Filho WP, Pimenta FP, Pereira GA, Pereira JA, Mattos-Guaraldi AL et al. Periodontal disease as reservoir for multi-resistant and hydrolytic enterobacterial species. Lett Appl Microbiol 2007;44:488-94.

33. Sedlacek MJ, Walter C. Antibiotic resistance in an in vitro subgingival biofilm model. Oral Microbiol Immunol 2007;22:333-39.

34. Diz P, Tomás I, Limeres J, Medina J, Fernández J, Alvarez M. Comparative efficacies of amoxicillin, clindamycin, and moxifloxacin in prevention of bacteremia following dental extractions. Antimicrob Agents Chemother 2006;50:2996-3002.

35. Gehanno P, Darantière $S$, Dubreuil C, Chobaut JC, Bobin S, Pages JC et al. A prospective, multicentre study of moxifloxacin concentrations in the sinus mucosa tissue of patients undergoing elective surgery of the sinus. J Antimicrob Chemother 2002; 49:821-6.

36. Arrieta JR, Galgano AS, Sakano E, Fonseca X, Amábile-Cuevas CF, Hernández-Oliva G et al. Moxifloxacin in Acute Sinusitis Study (MASS) Latin American Study Group.Moxifloxacin vs amoxicillin/ clavulanate in the treatment of acute sinusitis. Am J Otolaryngol 2007;28:78-82. 
37. Rakkar S, Roberts K, Towe BF, Flores SM, Heyd A, Warner J. Moxifloxacin versus amoxicillin clavulanate in the treatment of acute maxillary sinusitis: a primary care experience. Int J Clin Pract 2001;55:309-15.

38. Warnke PH, Becker ST, Springer IN, Haerle F, Ullmann U, Russo PA et al. Penicillin compared with other advanced broad spectrum antibiotics regarding antibacterial activity against oral pathogens isolated from odontogenic abscesses. J Craniomaxillofac Surg 2008 Aug 27 (Epub ahead of print).
39. Pfister W, Wegner K, Güntsch A, Jentsch H, Hoffmann T, Pick S. Moxifloxacin as an adjunctive antibiotic in treatment of periodontitis is effective in reduction of periodontopathogenic bacteria. Int J Med Microbiol 2007;297:93.

\section{CORRESPONDENCIA}

Carlos Martín Ardila Medina

Carrera 47 No. 20 sur 46

Envigado. Antioquía

cmartin@odontologia.udea.edu.co 